\title{
Do morphemes exist? On the role of morphology in language processing
}

\section{LAURA WINTHER BALLING}

This article provides an overview over the role of the morpheme in human language processing, on the basis of insights from theoretical morphology, psycholinguistics, and experimental investigations of word recognition in Danish. I describe how theoretical morphology assumes either morpheme- or word-based models, in spite of theoretical problems with both the morpheme and the word as units. Then, I consider two psycholinguistic traditions that focus on morphology: first, the so-called past-tense debate, between on the one hand models that distinguish sharply between regular and irregular words, and on the other hand models that postulate the same processing mechanism for all words irrespective of regularity; secondly, the broader word recognition literature which, in contrast to the past-tense debate, involves different types of morphology in different languages. Next, I summarise the results of a series of experiments on word recognition in Danish which generally indicate a functional role for morphology, but also show complex and interesting effects of morphological type, wholeword and morphological frequencies, and overlap in sound between a given word and the rest of the vocabulary. Finally, I summarise how morphology may be functional to language processing and indicate avenues of future research, with a critical view of traditional psycholinguistic methods and traditional theoretical units like the word and the morpheme. 